\title{
FOUR-COLOR OBSERVATIONS OF
}

\author{
BLUE HORIZONTAL-BRANCH STARS*
}

(Abstract)

\author{
A. G. DAVIS PHILIP \\ State University of New York at Albany and Dudley Observatory, New York, U.S.A.
}

Effective temperatures and surface gravities can be calculated for early-type stars with $0.5<\theta_{e}<0.7$ and $2.0<\log g<4.4$ by means of a grid computed by a graduate student at State University of New York at Albany, (Linda Matlock) which relates the four-color indices $b-y$ and $c_{1}$ to $\theta_{e}$ and $\log g$. The four-color indices must be dereddened and deblanketed, then $\theta_{e}$ and $\log g$ can be read off the grid with rms errors of \pm 0.015 in $\theta_{e}$ and \pm 0.2 in $\log g$. The details concerning these relations will be published soon in another article.

Blue horizontal-branch stars have been measured in the following globular clusters: M4, M5, M13, M55, and NGC 3201. Each star has been observed an average of four times and the rms error of the mean $c_{1}$ and $b-y$ indices are approximately \pm 0.03 to $\pm 0.04 \mathrm{mag}$. About ten blue horizontal-branch stars, well-spaced in $b-y$ color, are selected for measure in each globular cluster. The indices of the measured stars are plotted in Figure 1. The index $c_{1}$ is plotted against the index $b-y$ (both indices have been dereddened and deblanketed). Lines of equal $\theta_{e}$, from 0.5 to 0.7 and lines of equal $\log g$ from 2 to 4 are indicated. A double line indicates an evolutionary blue horizontal-branch model $\left(M_{c}=0.475 M_{0}, M=0.62 M_{0}, Y=0.3\right.$, and $\left.Z=10^{-3}\right)$ calculated by Sweigart and Gross (1973, unpublished). The indices of the blue horizontal-branch stars in the metal-poor globular cluster, M55, scatter about this double line and thus one could expect its parameters to agree with those defined by the blue horizontal branch model above. The indices of the blue horizontal-branch stars in the intermediate metal abundance $([\mathrm{Fe} / \mathrm{H}] \simeq-1.5)$ clusters $\mathrm{M} 13$ and NGC 3201 indicate surface gravities approximately 0.2 smaller in $\log g$ while the indices of the blue horizontal-branch stars in the more metal-rich $([\mathrm{Fe} / \mathrm{H}]=-0.6)$ clusters M4 and M5 indicate surface gravities approximately 0.2 larger in $\log g$. The bluest stars in the last two clusters scatter about M55 relation. It is not known which parameter, or combination of parameters, is causing this different behavior of blue horizontal-branch stars in the $c_{1}, b-y$ diagram or in its analogue, the $\log g, \log T_{\text {eff }}$ diagram, but it is evident from these data that at least one parameter must vary in some clusters.

\section{Acknowledgement}

The partial support of the National Science Foundation is acknowledged.

* Visiting astronomer, Kitt Peak National Observatory and Cerro Tololo Inter-American Observatory which are operated by the Association of Universities for Research in Astronomy, under contract with the National Science foundation. 


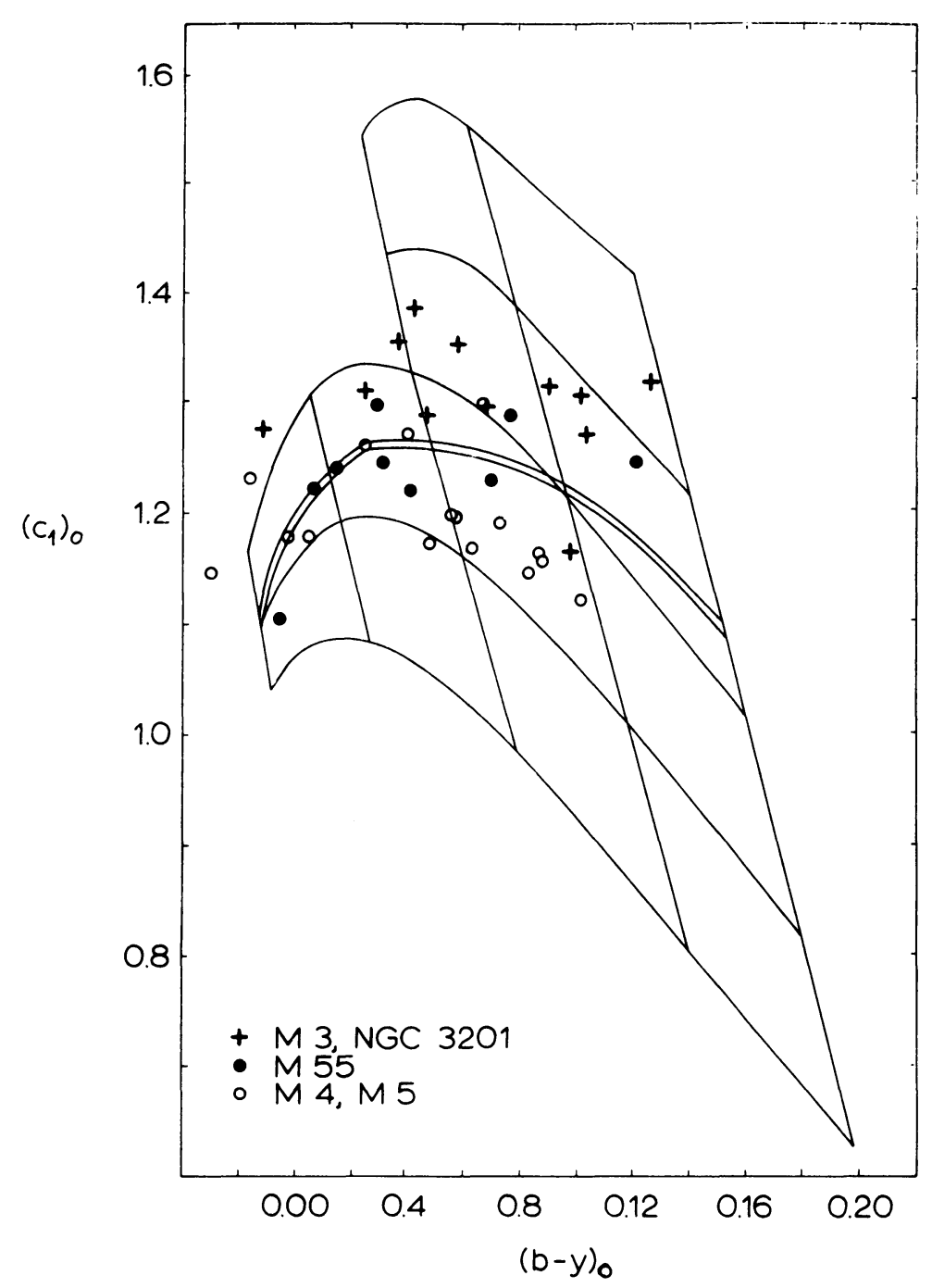

Fig. 1. Four-color observations of blue horizontal-branch stars. 